\title{
Validez y confiabilidad de la Escala Satisfacción Familiar en estudiantes universitarios de Lima Metropolitana, Perú
}

\author{
Validity and Reliability of the Family Satisfaction Scale in University \\ Students of Lima, Peru
}

\author{
David Villarreal-Zegarra ${ }^{1}$ \\ Angel Paz-Jesús ${ }^{3}$ \\ Instituto Peruano de Orientación Psicológica, Perú \\ Anthony Copez-Lonzoy ${ }^{2}$ \\ Universidad de San Martín de Porres, Perú \\ César Daniel Costa-Ball ${ }^{4}$ \\ Universidad Católica del Uruguay, Uruguay
}

Resumen. La presente investigación tuvo como objetivo analizar las propiedades psicométricas de la Escala de Satisfacción Familiar (FSS) en una muestra de 607 estudiantes de una universidad privada en Lima Metropolitana, con edades que oscilan entre 16 a 28 años. Se tradujo la escala con el método de traducción inversa; se realizaron análisis factorial exploratorio y confirmatorio, identificando valores óptimos para una solución unifactorial. Asimismo, se analizó la relación con la variable comunicación familiar, que señala una correlación positiva, directa y con un tamaño del efecto moderado. En el análisis de invarianza según sexo, se encontró un nivel de invarianza débil. Finalmente, se reportan valores óptimos de confiabilidad. Se concluye que la FSS presenta evidencias de validez y confiabilidad, recomendando su uso.

Palabras clave Satisfacción familiar, modelo circumplejo, confiabilidad, análisis factorial, invarianza.

Abstract The present study is an instrumental research that aims to analyze the psychometric properties of the family satisfaction scale (FSS) in a sample of 607 students from a private university in Lima Metropolitana, aged between 16 and 28 years old. The scale was translated with the inverse method. An exploratory and confirmatory factor analysis was performed, identifying optimal values for a unifactorial solution. The relationship with the family communication variable was analyzed in a positive, direct and moderated effect size. In the analysis of invariance according to sex, it indicated a weak level of invariance. Finally, optimal values of reliability are reported. It is concluded that the FSS presents evidence of validity and reliability, its use being recommended.

Keywords Family satisfaction, Circumplex model, Reliability, Factorial analysis, Multigroup analysis.

\footnotetext{
${ }^{1}$ David Villarreal-Zegarra. Instituto Peruano de Orientación Psicológica, Perú. Dirección postal: IPOPS Jr. Manuel Corpancho 208, Lima Perú. E-mail: davidvillarreal@ipops.pe.

${ }^{2}$ Anthony Copez-Lonzoy. Universidad de San Martín de Porres, Perú. E-mail: anthonycopez@yahoo.es

${ }^{3}$ Angel Paz-Jesús. Instituto Peruano de Orientación Psicológica, Perú. E-mail: angelpazjesus@ipops.pe

${ }^{4}$ César Daniel Costa-Ball. Universidad Católica del Uruguay, Uruguay. E-mail: ccosta@ucu.edu.uy
} 


\section{Introducción}

El desempeño académico eficaz de los jóvenes que se encuentran cursando estudios universitarios obtiene gran atención por parte de los entes formadores, esto debido a su estrecha relación con la deserción académica (Martinis-Mercado, Cardozo, Abraham, González \& Sarmiento, 2016), la motivación (Stover, de la Iglesia, Boubeta \& Liporace, 2012), entre otros. Diversos estudios sobre la influencia de las relaciones familiares en el desempeño académico universitario hacen notar la importancia del soporte familiar en estos jóvenes (Mushtaq \& Nawaz, 2012; Stallman, 2010; Stephens, Fryberg, Markus, Johnson \& Covarrubias, 2012). De manera que resulta necesario contar con herramientas de evaluación que permitan identificar este tipo de relaciones familiares.

Una adecuada evaluación del sistema familiar implica, en parte, contar con instrumentos con suficientes evidencias de validez y confiabilidad; esto comprende una limitación en contexto actual, debido al reducido número de escalas que cumplen con estas propiedades (Instituto Nacional de Salud Mental, 2008).

Dentro de las variables más relevantes en el estudio de las relaciones familiares se encuentra la satisfacción familiar, puesto que investigaciones realizadas con jóvenes universitarios señalan su relación con la depresión (Álvarez, López, Mendoza \& Bravo, 2013), los conflictos que se generan en el hogar por la actividad laboral de los padres (Turliuc \& Buliga 2014), la calidad de vida (Bernal, Arocena \& Ceballos, 2011), entre otros.

La satisfacción familiar comprende una respuesta subjetiva de agrado, asociada con la cohesión de la familia, la flexibilidad al cambio y la comunicación entre sus miembros (Olson, Gorall \& Tiesel, 2006; Sanz, 2008). Entre los instrumentos para medir este constructo, se encuentra la Escala de Satisfacción Familiar o FSS (por sus siglas en inglés), elaborada originalmente por Olson y Wilson (1982) en población estadounidense y basada en el modelo circumplejo de los sistemas de pareja y familia.

El modelo de Olson plantea que las relaciones familiares se centran en tres elementos: cohesión, flexibilidad y comunicación (elemento transversal dentro de la relación). En este modelo, la satisfacción familiar cumple la función de indicador global respecto al agrado/desagrado sobre cada una las tres variables (Olson et al., 2006).

Originalmente, en el estudio del FSS (14 ítems), se realizó un análisis de componentes principales usando la rotación varimax; arrojó dos factores: 8 ítems que evalúan la vinculación emocional (cohesión familiar) y 6 ítems dirigidos a evaluar la satisfacción hacia la flexibilidad. Los índices de confiabilidad fueron adecuados para las dimensiones de cohesión $(\alpha=.85)$, adaptabilidad $(\alpha=$ .84) y la escala total $(\alpha=.92)$. Asimismo, los valores del test-retest resultaron óptimos (Olson \& Wilson, 1982).

La evolución misma del modelo teórico permitió desarrollar una nueva versión del FSS. Actualmente, se cuenta con mejoras significativas; entre ellas, la consolidación de la unidimensionalidad; inclusión de ítems para evaluar la comunicación familiar; evidencias de estructura interna; evidencias basadas en relaciones con otras variables como calidad de vida $(r=.67$; r-squared $=.45)$, satisfacción marital $(r=.70 ; \mathrm{r}$-squared $=.49)$; así como niveles de confiabilidad adecuados $(\alpha$ $=.92 ; n=2465)$ (Olson et al., 2006).

En Grecia se realizó un estudio con 584 participantes (32.4\% hombres y 67.6\% mujeres). Para análisis de confiabilidad se utilizó el índice de consistencia interna alfa de Cronbach $(\alpha=.90)$; la estabilidad por medio de test-retest fue adecuada (.98), con una relación estadísticamente significativa $(r=.78, p<.001)$ con la escala de comunicación familiar (Koutra, Triliva, Roumeliotaki, Lionis \& Vgotzas, 2012). Asimismo, en Malasia se confirmó la unidimensionalidad de la escala a través de un análisis factorial confirmatorio (AFC) que presentó índices de ajuste adecuados para una sola dimensión (RMSE $A=.06, C F I=.94, N F I=.94, T L I$ $=.93)$ y niveles de confiabilidad óptimos $(\alpha=.94)$ (Aazami, Shamsuddin \& Akmal, 2015).

En el contexto latinoamericano, el FSS ha sido utilizado en Uruguay, donde replicó parcialmente la unidimensional propuesta por Olson et al. (2006). En este estudio se utilizó el análisis factorial exploratorio $\left(K M O=.89 ; X^{2}\right.$ 
$=1078.175 ; g l=45 ; p<.001)$ mediante el método de extracción de componentes principales y análisis paralelo. Se extrajo el 47\% de la varianza (Costa-Ball, Tornaría, del Luján, del Arca, Masjuan \& Olson, 2013).

En el Perú, aún no se reportan investigaciones sobre la nueva versión del FSS, lo cual limita los estudios y evaluaciones de la satisfacción familiar. Por lo tanto, ante la ausencia de adecuados instrumentos para medir esta variable en contexto universitario, se propone como objetivo analizar las propiedades psicométricas de la escala de satisfacción familiar en estudiantes universitarios.

\section{Método}

\section{Diseño}

La presente es una investigación instrumental (Montero \& León, 2002; Ato, López \& Benavente, 2013), cuyo objetivo es la adaptación psicométrica de la escala satisfacción familiar (Olson et al., 2006).

\section{Participantes}

La muestra consta de 607 estudiantes universitarios de una universidad privada en Lima Metropolitana, Perú; varones $(n=169,27.8 \%)$ y mujeres $(n=438$, $72.2 \%)$, con edades comprendidas entre 16 a 28 años $(M=19.8, D T=3.1)$. El $61.8 \%$ solo estudian $(n=375)$ y el $38.2 \%$ trabajaba y estudia $(n=232)$. Además, el $92 \%$ son solteros que nunca se han casado ni han convivido $(n=559)$.

Los criterios de inclusión planteados fueron: ser estudiante matriculado en el presente ciclo académico, pertenecer al rango de edades comprendidas entre 16 a 28 años. Criterio de exclusión: Tener más de 28 años, estar casado, conviviendo o haberse divorciado.

\section{Instrumentos}

Escala de Satisfacción Familiar - FSS (Olson et al., 2006)

Diseñada para evaluar la satisfacción que perciben los miembros sobre el funcionamiento familiar; implica los niveles de cercanía emocional, la capacidad para adaptarse a los cambios, la calidad de la comunicación y la forma de resolver los problemas. Consta de diez ítems de tipo Likert de cinco alternativas, cuya valoración es 1 (extremadamente insatisfecho), 2 (generalmente insatisfecho), 3 (indeciso), 4 (generalmente satisfecho) y 5 (extremadamente satisfecho), con una puntuación máxima posible de 50 y mínima de 10 . En la versión original se obtuvieron valores de consistencia interna $(n=2465, \alpha=.92)$ y estabilidad mediante test-retest (.85) adecuados, siendo representativas de la población estadounidense $(M=37.5, D E=8.5)$.

Escala de Comunicación Familiar - FCS (Olson et al., 2006)

Consta de 10 ítems de tipo Likert, con cinco alternativas con valoraciones de 1 (extremadamente insatisfecho), 2 (generalmente insatisfecho), 3 (indeciso), 4 (generalmente satisfecho) y 5 (extremadamente satisfecho). Las puntuaciones varían entre 10 a 50, la versión original representa a la población estadounidense $(M=$ $36.2, D T=9.0, \alpha=.90)$ y en la versión para estudiantes universitarios en Lima, se confirma la unidimensionalidad con adecuadas propiedades psicométricas $\chi^{2}=$ 80.31; $\chi^{2} / \mathrm{gl}=2.36 ; C F I=1.00 ; G F I=.995 ; A G F I=$ $.992 ; \mathrm{RMSE} A=.001 ; \mathrm{RMR}=.050(n=245)($ CopezLonzoy, Villarreal-Zegarra \& Paz-Jesús, 2016).

\section{Procedimiento}

En primer lugar, se llevó a cabo la traducción al español de los ítems de la FSS, para lo cual se tuvieron presentes las directrices de la Comisión Internacional de Tests (ITC) sobre la adaptación de los tests de una cultura a otra (Muñiz, Elosua \& Hambleton, 2013). Asimismo, se utilizó el método de traducción back translation (Hambleton, 1996), el cual consistió en traducir la escala del inglés al español y, posteriormente, retraducir la versión del español al inglés, asegurando la equivalencia de la traducción. Se contó con traductores independientes con estudios de psicología, quienes fueron instruidos acerca del test, el objetivo de su adaptación, el constructo medido, el formato de respuesta y la forma de corrección.

La recolección de los datos se realizó de forma colectiva, informando a los participantes de forma oral sobre el objetivo de la investigación y la 
confidencialidad de los datos. Además, los participantes firmaron un consentimiento informado, accediendo ser parte del estudio.

\section{Análisis de datos}

Para los análisis, se utilizaron los programas estadísticos FACTOR 10.4.01 (Lorenzo-Seva \& Ferrando, 2007) para el análisis factorial exploratorio y de confiabilidad (AFE); el programa EQS 6.1 para el análisis factorial confirmatorio, analizar la invarianza, descriptivo y SPSS 22 para el análisis de correlación.

Preliminarmente, se analizaron las medidas de tendencia central y dispersión, se analizó la mulicolinealidad mediante indicadores de asimetría y curtosis (Pérez \& Medrano, 2010; Ferrando \& Anguiano-Carrasco, 2010), normalidad multivariante con la prueba de Mardia (1970) y la discriminación se examinó mediante la correlación ítem-test (Elosua, 2004; Kline, 1986).

El análisis factorial exploratorio (AFE) y el análisis factorial confirmatorio (AFC) se realizaron dividendo la muestra en dos grupos (Lloret-Segura, FerreresTraver, Hernández-Baeza \& Tomás-Marco, 2014); el primero para el AFE y el segundo para el AFC. Previo al AFE se analizó la medida de adecuación muestral KMO, el determinante y la esfericidad de Bartlett (Pardo \& Ruiz, 2002; Hair, Anderson, Tatham \& Black, 1999). Por la naturaleza de los datos, se utilizaron matrices policóricas (Domínguez, 2014), el método de extracción utilizado es el de mínimos cuadrados no ponderados (Hair et al., 1999). Para determinar el número de factores a retener, se utilizó el análisis paralelo (AP), por considerarse el más adecuado para realizar análisis factorial (Timmerman \& Lorenzo-Seva, 2011).

En el análisis factorial confirmatorio (AFC), se utilizó el método de estimación de mínimos cuadrados no ponderados (Lloret-Segura, et al., 2014), junto con una serie de indicadores de bondad ajuste, entre ellos $X^{2}, X^{2} / g l, C F I, R M S E A$, SRMR y PSRMR (Ferrando \& Anguiano-Carrasco, 2010; Hair et al., 1999; Hu \& Bentler, 1998; Lloret-Segura, et al., 2014; Manzano \& Zamora, 2010; Ruiz, Pardo \& San Martín, 2010).

La relación con la escala de comunicación familiar
(Copez-Lonzoy et al., 2016) se evaluó seleccionando un grupo aleatorio de 220 participantes. Se analizó la normalidad, la correlación y el tamaño del efecto. Asimismo, se analizó la invarianza del modelo (Mellenbergh, 1989; Meredith, 1993) a través de la selección de dos grupos equivalentes por sexo y el establecimiento de tres modelos con restricciones progresivas (Byrne, 1994; Widaman \& Reise, 1997). Finalmente, para el análisis de confiabilidad, se utilizaron diferentes indicadores de consistencia interna.

\section{Resultados}

\section{Análisis preliminar de los ítems}

Los hallazgos descriptivos $(M=34.79, D T=8.33$, $n=607$, Min $=10$, Max $=50)$ sugieren descartar la presencia de multicolinealidad (véase tabla 1). Asimismo, la correlación ítem-test resultó óptima para todos los ítems (DeVellis, 2012; Kline, 1986). Los indicadores de asimetría y curtosis resultaron adecuados, dentro del rango + / - 1.5 (Pérez \& Medrano, 2010; Ferrando \& Anguiano-Carrasco, 2010) (Tabla 1).

El análisis de Mardia (1970) para la asimetría y curtosis multivariante señala un coeficiente de asimetría de 8.279, $g l=220, p=1.00$ y un coeficiente de curtosis de 161.754, $p<.001$, lo que señala la ausencia de una distribución de normalidad multivariante en los datos.

Se dividió la muestra en dos grupos aleatorizados y proporcionales. En el primero, se aplicó AFE $(n=303)$ $\mathrm{y}$ al segundo AFC $(n=304)$ (Lloret-Segura et al., 2014).

\section{Análisis Factorial}

El análisis previo a la aplicación del AFE indicó una matriz de la determinante de .008. La medida de adecuación muestral KMO o Kaiser-Meyer-Olkin, que contrasta si las correlaciones parciales entre las variables son adecuadas, obtuvo un valor de .92 considerado como óptimo (Pardo \& Ruiz, 2002; Hair et al., 1999). Asimismo, la prueba de esfericidad de Bartlett evidenció suficiente intercorrelación entre los items, $\chi^{2}(45)=1429.8, p$ $<.001, n=303$, por lo que se procedió con el análisis factorial exploratorio.

En tanto, el método de extracción utilizado fue el 
Tabla 1

Media, desviación estándar, asimetría, curtosis de los items y la escala total de la escala de satisfacción familiar $(n=607)$

\begin{tabular}{cccccccccc}
\hline Ítem & $M$ & $I C 95 \%$ & $D T$ & $g^{1}$ & $S S I$ & $g^{2}$ & $r_{i t c}$ & $\%$ Min & $\%$ Max \\
Ítem 1 & 3.65 & {$[3.53 ; 3.77]$} & 1.12 & -0.7 & -0.28 & -0.22 & .742 & 5.6 & 23.7 \\
Ítem 2 & 3.22 & {$[3.11 ; 3.33]$} & 1.07 & -0.25 & -0.11 & -0.61 & .661 & 6.3 & 10 \\
Ítem 3 & 3.45 & {$[3.34 ; 3.56]$} & 1.08 & -0.48 & -0.21 & -0.43 & .642 & 5.3 & 15.3 \\
Ítem 4 & 3.89 & {$[3.78 ; 3.99]$} & 1.04 & -0.83 & -0.38 & 0.06 & .692 & 2.6 & 32.1 \\
Ítem 5 & 3.63 & {$[3.51 ; 3.75]$} & 1.13 & -0.5 & -0.2 & -0.58 & .713 & 4.1 & 25.9 \\
Ítem 6 & 3.53 & {$[3.41 ; 3.65]$} & 1.14 & -0.52 & -0.2 & -0.55 & .746 & 5.6 & 20.6 \\
Ítem 7 & 3.36 & {$[3.24 ; 3.49]$} & 1.22 & -0.34 & -0.11 & -0.89 & .629 & 8.2 & 19.9 \\
Ítem 8 & 3.1 & {$[2.98 ; 3.22]$} & 1.17 & -0.16 & -0.06 & -0.9 & .629 & 10 & 10.9 \\
Ítem 9 & 3.1 & {$[2.98 ; 3.21]$} & 1.11 & -0.18 & -0.07 & -0.64 & .63 & 9.4 & 9.7 \\
Ítem 10 & 3.88 & {$[3.76 ; 3.99]$} & 1.12 & -0.9 & -0.36 & 0.08 & .655 & 4.4 & 35.1 \\
\hline
\end{tabular}

Nota. $M=$ media; $D T=$ desviación típica; $g^{1}=$ asimetría; $g^{2}=$ curtosis; $S S I=$ índice estandarizado de asimetría; $r_{i t c}=$ correlación inter ítem promedio

de mínimos cuadrados no ponderados o ULS (Hair et al., 1999). Se utilizó el análisis paralelo óptimo (Timmerman \& Lorenzo-Seva, 2011) para determinar el número de factores a retener; se encontró una solución unifactorial, por lo que no se realizó rotación (Pérez \& Medrano, 2010).

La varianza promedio extraída (AVE) se evaluó con el criterio de $>.50$ (Fornell \& Larker, 1981) para la validez interna de los ítems. Los pesos factoriales fluctuaron entre .621 a .810 (ver tabla 2) y la varianza explicada fue de $56.2 \%$, considerándose aceptable (Lloret-Segura et al., 2014).

En coherencia con el AFE, se realizó el AFC con base en el modelo de un solo factor mediante el método de estimación de mínimos cuadrados no ponderados (Lloret-Segura, et al., 2014). Se estimó el ajuste del modelo con base en diferentes índices, entre ellos, el CFI. Se consideran óptimos valores mayores a .95 (Hu \& Bentler, 1998); RMSEA y el SRMR, cuyos puntajes óptimos son por debajo de .05 (Lloret-Segura, et al., 2014). Los valores obtenidos indican un ajuste adecuado para el modelo
Tabla 2

Cargas factoriales e indices de bondad de ajuste en el análisis factorial exploratorio $(n=303)$ para los items de la Escala de Satisfacción Familiar

\begin{tabular}{ccc}
\hline & \multicolumn{2}{c}{ Modelo de un factor } \\
& $F 1$ & $h 2$ \\
\hline Ítem 1 & .810 & .656 \\
Ítem 2 & .627 & .394 \\
Ítem 3 & .621 & .386 \\
Ítem 4 & .784 & .614 \\
Ítem 5 & .761 & .579 \\
Ítem 6 & .766 & .587 \\
Ítem 7 & .720 & .519 \\
Ítem 8 & .671 & .451 \\
Ítem 9 & .661 & .437 \\
Ítem 10 & .731 & .534 \\
AVE & & .554
\end{tabular}

Nota. AVE $=$ varianza extraída promedio; $\mathrm{h} 2=$ comunilidad 
unidimensional $X_{(34)}^{2}=115.31 ; p<.001 ; X^{2} / g l=3.39$; $C F I=.985 ; \operatorname{RMSE} A=.048 ;$ SRMR $=.043 ; n=304$.

\section{Evidencias basadas en relaciones con otras variables}

El modelo teórico de Olson (2006) asume una relación directa y positiva entre la satisfacción y la comunicación familiar, por lo que se procedió a contrastar esta hipótesis. El análisis de la normalidad mostró una distribución no paramétrica, tanto para satisfacción familiar $S W_{(220)}=.986, p=.033$ como para comunicación familiar $S W(220)=.974, p<.001$. Por lo anterior, se utilizó el estadístico de Spearman para analizar la correlación y se tomó la medida del coeficiente de determinación para evaluar el tamaño del efecto $r_{\mathrm{s}}=.707, p<.001, n=220, r^{2}=.499$. Se encontró una correlación significativa y un tamaño del efecto moderado (Cohen, 1992).

\section{Invarianza de medición}

Se compararon dos grupos equivalentes de varones $(n=169)$ y mujeres $(n=169$, seleccionadas de forma aleatoria), para analizar la invarianza. Se utilizó el método de Máxima verosimilitud. Se establecieron tres modelos con restricciones progresivas (Byrne, 1994; Milfont \& Fischer, 2015; Widaman \& Reise, 1997). Además, se tuvo en cuenta el cumplimiento de diferentes niveles de invarianza configuracional, invarianza métrica e invarianza escalar.

Los modelos se compararon con base al Chicuadrado $\left(\chi^{2}\right)$, grados de libertad $(g)$, Chi cuadrado sobre grados de libertad $\left(\chi^{2} / g\right)$ e indicadores como CFI, TLI y RMSE $A$ con IC al $90 \%$ (ver tabla 3 ). Se evidencian diferencias entre el modelo configural y métrico, así como entre el modelo métrico y el escalar.
Se encontró una invarianza factorial débil entre los grupos analizados (Byrne, 1994); es decir, que los puntajes de varones y mujeres no son equivalentes.

\section{Análisis de confiabilidad}

La confiabilidad se estimó con base en la muestra total $(\mathrm{n}=607)$ mediante el coeficiente alfa, de $\alpha=.910$ [IC 95\% .895 - .923] para puntajes observables, y otros coeficientes para variables latentes como $\omega=.925 \mathrm{y} \mathrm{H}$ $=.929$. Se obtuvieron valores óptimos en todos casos (Hancock \& Mueller, 2001; McDonald, 2013; Oviedo \& Campo-Arias, 2005).

\section{Discusión}

Los estudios con población universitaria son de suma importancia para diferentes ámbitos de investigación (Baader et al., 2014; Barra, 2010; Caballero, González \& Palacio, 2015; Becerra-González \& Reidl, 2015), ya que corresponden a los futuros profesionales de una nación. Esto cobra mayor relevancia en el contexto donde la familia mantiene una gran influencia en los jóvenes (Llamas \& Morgan, 2012). De manera que el presente estudio buscó aportar una nueva herramienta de evaluación familiar en estudiantes universitarios.

El instrumento se tradujo con el fin de establecer la equivalencia conceptual y semántica de la versión final con la escala original a través de la estrategia de back translation o traducción inversa (Hambleton, 1996). Para procurar que este proceso se realice de forma adecuada, se siguieron las directrices de la ITC o comisión internacional de test (Muñiz et al., 2013). Se encontró que la versión original y la versión retrotraducida eran equivalentes, por lo que la versión traducida al español, presenta equivalencia conceptual

Tabla 3

Invarianza de medición según sexo con muestras equivalentes usando máxima verosimilitud

\begin{tabular}{ccccccc}
\hline Modelos & $X^{2}[g l$ & $X^{2} / g l$ & $R M S E A[I C 90 \%](\Delta$ RMSEA) & TLI $(\Delta T L I)$ & $C F I(\Delta C F I)$ & $p($ comparación) \\
\hline Configural & $150.065[70]$ & 2.144 & $.058[.045-.071]$ & .937 & .951 & - \\
Métrica & $174.814[79]$ & 2.213 & $.060[.048-.072](.002)$ & $.933(.004)$ & $.941(.010)$ & .003 (M1 vs M2) \\
Escalar & $188.354[89]$ & 2.116 & $.058[.046-.069](.001)$ & $.938(.002)$ & $.939(.012)$ & .005 (M2 vs M3) \\
\hline
\end{tabular}

Nota. varones $(n=169)$ y mujeres $(n=169)$. 
y semántica con la versión original. Esto se apoya en los resultados de Copez-Lonzoy et al. (2016), en la escala de comunicación familiar de Olson et al. (2016), donde se encuentra equivalencia entre las dos versiones.

Para el AFE, el análisis paralelo (Timmerman \& Lorenzo-Seva, 2011) sugiere una solución unifactorial que resulta estable a nivel de cargas factoriales y número de ítems (Ferrando y Lorenzo-Seva, 2014). Con base en esta se realizó un AFC en el que se identificaron índices de bondad de ajuste óptimos en la solución unidimensional (Hu \& Bentler, 1998; Lloret-Segura, et al., 2014). Los análisis utilizados para estudiar la estructura interna señalaron un adecuado ajuste para la solución unidimensional, apoyando la propuesta original de Olson et al. (2006) y concordando con lo reportado por otros autores (Aazami et al., 2015; Costa-Ball et al., 2013).

Dentro del modelo circumplejo, se plantean hipótesis sobre los sistemas familiares, una de ellas es la relación entre satisfacción y comunicación familiar (Olson, 2011, 2000; Olson \& Gorall, 2006). Esta se evaluó con la FSS con la FCS (Copez-Lonzoy et al., 2016; Olson et al., 2006), donde se halla una correlación positiva, directa y con un efecto moderado $r_{s}=.707, p<.001$, $n=220, r^{2}=.499$, que apoya la hipótesis del modelo. Asimismo, los resultados concuerdan con lo reportado por Sobrino (2008), que utilizó las versiones más antiguas de ambas escalas en estudiantes universitarios de Lima, apoyando la hipótesis de la relación de que la comunicación se relaciona con la satisfacción familiar, como propone el modelo circumplejo.

El análisis de invarianza identificó que las puntuaciones entre hombres y mujeres no son equivalentes; se encontró una invarianza débil (Byrne, 1994). Lo anterior indica que, en la muestra evaluada, el sexo juega un papel importante en la percepción subjetiva de la satisfacción familiar, como refieren Rocha-Sánchez y Díaz-Loving (2005).

Finalmente, se estimó la confiabilidad a través de diferentes índices de bondad de ajuste para puntajes observables y latentes, encontrando valores óptimos en todos los casos (Berge \& Socan, 2004; DeVellis,
2012; Elosua y Zumbo, 2008; McDonald, 1999; Oviedo \& Campo-Arias, 2005). Esto concuerda con lo propuesto por otros autores (Aazami et al., 2015; Costa-Ball et al., 2013; Koutra et al., 2012; Olson et al., 2006), permitiendo inferir que estos resultados son confiables para la FSS.

Se concluye que la Escala de Satisfacción Familiar es un instrumento con evidencias de validez y confiabilidad, que posee equivalencia lingüística-conceptual con la escala original (inglés), adecuados índices de bondad de ajuste para el modelo unidimensional, óptimos índices de confiabilidad e invarianza factorial débil según sexo. Asimismo, se relaciona directa y positivamente con la comunicación familiar, apoyando el modelo circumplejo de los sistemas de pareja y familia. Es necesario tener presente que el muestreo de este estudio fue de tipo incidental por lo que la generalización de los resultados es una limitación.

Se recomienda poder realizar investigaciones con el FSS en diferentes contextos, para analizar las propiedades psicométricas del mismo (escolares, adultos, población clínica, entre otros). Además, sería oportuno realizar estudios más detallados sobre la equivalencia de los puntajes entre varones y mujeres; y desarrollar baremos con base en muestreos probabilísticos.

\section{Referencias}

Aazami, S., Shamsuddin, K., \& Akmal, S. (2015). Malay version of the family satisfaction scale: validity and reliability among malaysian working women. International Journal of Public Health Research, 5(2), 606-612.

Álvarez, Y. S., López, M. V., Mendoza, O. M., \& Bravo, G. H. (2013). Relación entre satisfacción con el ambiente familiar y depresión en adolescentes. Psicología y salud, 23(1), 141-148.

Ato, M., López, J., \& Benavente, A. (2013). Un sistema de clasificación de los diseños de investigación en psicología. Anales en psicología, 29(3), 1038-1059. doi:10.6018/analesps.29.3.178511

Baader, T., Rojas, C., Molina, J. L., Gotelli, M., Alamo, Fierro, C., ... Dittus, P. (2014). Diagnóstico de la 
prevalencia de trastornos de la salud mental en estudiantes universitarios y los factores de riesgo emocionales asociados. Revista chilena de neuropsiquiatría, 52(3), 167-176. doi: 10.4067/S071792272014000300004

Barra, E. (2010). Bienestar psicológico y orientación de rol sexual en estudiantes universitarios. Terapia psicológica, 28(1), 119-125. doi: 10.4067/S071848082010000100011

Becerra-González, C. E., \& Reidl, L. M. (2015). Motivación, autoeficacia, estilo atribucional y rendimiento escolar de estudiantes de bachillerato. Revista Electrónica de Investigación Educativa, 17(3), 79-93.

Berge, J. M., \& Socan, G. (2004). The greatest lower bound to the reliability of a test and the hypothesis of unidimensionality. Psychometrika, 69(4), 613-625.

Bernal, A. C. A. L., Arocena, F. A. L., \& Ceballos, J. C. M. (2011). Bienestar subjetivo y satisfacción con la vida de familia en adolescentes mexicanos de bachillerato. Psicología Iberoamericana, 19(2), 17-26.

Byrne, B. M. (1994). Structural equation modeling with EQS and EQS/Windows. Thousand Oaks, CA: Sage Publications.

Caballero, C., González, O., \& Palacio, J. (2015). Relationship between burnout and engagement, with depression, anxiety and academic performance in university students. Revista Salud Uninorte, 31(1), 59-69. doi: 10.14482/sun.30.1.4309

Cohen, J. (1992). A power primer. Psychological bulletin, 112(1), 155-159.

Copez-Lonzoy, A., Villarreal-Zegarra, D., \& Paz-Jesús, Á. (2016). Propiedades psicométricas de la Escala de Comunicación Familiar en estudiantes universitarios. Revista Costarricense de Psicología, 35(1), 37-52.

Costa-Ball, D., Tornaría, G., del Luján, M., del Arca, D., Masjuan, N., \& Olson, D. H. (2013). Propiedades Psicométricas del FACES IV: Estudio de validez en población uruguaya. Ciencias Psicológicas, 7(2), 119-132.

DeVellis, R. F. (2012). Scale Development, Theory and Applicatons. Los Angeles: SAGE.
Domínguez, S. (2014). ¿Matrices Policóricas/Tetracóricas o Matrices Pearson? Un estudio metodológico. Revista Argentina de Ciencias del Comportamiento, 6(1), 39-48.

Elosua, P. (2004). Sobre la validez de los tests. Psicothema, 15(2), 315-321.

Elosua, P., \& Zumbo, B. (2008). Coeficientes de fiabilidad para escalas de respuesta categórica ordenada. Psicothema, 20(4), 896-901.

Ferrando, P. J., \& Anguiano-Carrasco, C. (2010). El análisis factorial como técnica de investigación en psicología. Papeles del Psicólogo, 31(1), 18-33.

Ferrando, P. J., \& Lorenzo-Seva, U. (2014). El análisis factorial exploratorio de los ítems: algunas consideraciones adicionales. Anales de psicología, 30(3), 1170-1175.

Fornell, C., \& Larcker, D. F. (1981). Evaluating structural equation models with unobservable variables and measurement error. Journal of Marketing Research, $28,39-50$.

Hair, J.F.; Anderson, R.E.; Tatham, R.L. y Black, W. (1999). Análisis Multivariante. Madrid: Prentice Hall.

Hambleton, R. K. (1996). Adaptación de tests para su uso en diferentes idiomas y culturas: fuentes de error, posibles soluciones y directrices prácticas. En J. Muñiz (Coor.), Psicometría. Madrid: Universitas.

Hancock, G., \& Mueller, R. (2001). Rethinking construct reliability within latent variable systems. Structural equation modeling: Present and future, 195-216.

Hu, L., \& Bentler, P. (1998). Fit indices in covariance structure modeling: Sensivity to underparameterized model misspecification. Psychological Methods, 3(4), 424-453.

Instituto Nacional de Salud Mental. (2008). Base de Datos de instrumentos de evaluación de Salud Mental y Psiquiatría. Anales de Salud Mental, 24(1).

Kline, P. (1986). A handbook oftest construction: Introduction to psychometric design. New York: Methuen.

Koutra, K., Triliva, S., Roumeliotaki, T., Lionis, C., \& Vgotzas, A. (2012). Cross cultural adaptation and validation of the greek version of the family 
adaptabity and cohesion evaluation scales IV package - FACES IV package. Journal of Family Issues, 34(12), 1647-1672. doi: 10.1177/0192513X12462818

Llamas, J. D., \& Morgan, M. (2012) . The Importance of Familia for Latina/o College Students: Examining the Role of Familial Support in Intragroup Marginalization. Cultural Diversity and Ethnic Minority Psychology, 18(4), 395- 403. doi: 10.1037 / a0029756

Lloret-Segura, S., Ferreres-Traver, A., HernándezBaeza, A., \& Tomás-Marco, I. (2014). El Análisis Factorial Exploratorio de los Ítems: una guía práctica, revisada y actualizada. Anales de psicología, 30(3), 1151-1169. doi: / 10.6018/analesps.30.3.199361

Lorenzo-Seva, U., \& Ferrando, P. (2007). FACTOR: $A$ computer program to fit the exploratory factor analysis model. University Rovira i Virgili.

Manzano, A., \& Zamora, S. (2010). Sistema de ecuaciones estructurales: una herramienta de investigación. México D.F.: Centro Nacional de Evaluación para la Educación Superior.

Mardia, K. V. (1970). Measures of multivariate skewnees and kurtosis with applications. Biometrika, 57, 519-530.

Martinis-Mercado, D., Cardozo, S., Abraham, M., González, C., \& Sarmiento, R. (2016). Study of desertion causal factors in veterinary sciences students. Revista Veterinaria, 27(2), 130-133.

McDonald, R. P. (1999). Test theory: A unified approach. Mahwah, NJ: Erlbaum.

Mellenbergh, G. (1989). Item bias and item response theory. International Journal of Mathematical and Statistical Psychology, 24, 49-82.

Meredith, W. (1993). Measurement invariance, fator analysis and factorial invariance. Psychometrika, 58(4), 525-543.

Milfont, T. L., \& Fischer, R. (2015). Testing measurement invariance across groups: Applications in cross-cultural research. International Journal of psychological research, 3(1), 111-130.
Montero, I., \& León, O. (2002). Clasificación y descripción de las metodologías de investigación en Psicología. International Journal of Clinical and Health Psychology, 2(3), 503-508.

Muñiz, J., Elosua, P., \& Hambleton, R. (2013). Directrices para la traducción y adaptación de los tests: segunda edición. Psicothema, 25(2), 151-157.

Mushtaq, I., \& Nawaz, S. (2012). Factors affecting students' academic performance. Global Journal of Management and Business Research, 12(9), 17-22.

Olson, D. H. (2000). Circumplex Model of Marital and Family Systems. Journal of Family Therapy, 22(2), 144-167.

Olson, D. (2011). Faces IV and the circumplex model: Validation study. Journal of marital and family therapy, 37(1). 64-80.

Olson, D., \& Gorall, D. (2006). FACES IV \& the Circumplex Model. Minnesota: Life Innovations.

Olson, D., Gorall, D., \& Tiesel, J. (2006). FACES IV Package. Administration Manual. Minnesota: Life Innovations.

Olson, D., \& Wilson, L. R. (1982). Family Satisfaction Scale. En D.H. Olson, H.I. McCubbin, H. Barnes, A. Larsen, M. Muxen \& M. Wilson (eds.), Family inventories: Inventories used in a national survey of families across the family life cycle, 43-49.

St. Paul, MN: University of Minnesota.

Oviedo, H., \& Campo-Arias, A. (2005). Aproximación al uso del coeficiente alfa de Cronbach. Revista colombiana de psiquiatría, 34(4), 572-580.

Pardo, A., \& Ruiz, M. (2002). SPSS 11 Guía para el análisis de datos. Madrid: Mc Graw - Hill.

Pérez, E., \& Medrano, L. (2010). Análisis Factorial Exploratorio: Bases Conceptuales y Metodológicas. Revista Argentina de Ciencias del Comportamiento, 2(1), 58-66.

Rocha-Sánchez, T., \& Díaz-Loving, R. (2005). Cultura de género: La brecha ideológica entre hombres y mujeres. Anales de Psicología, 21(1), 42-49.

Ruiz, M., Pardo, A., \& San Martín, R. (2010). Modelos 
de Ecuaciones Estructurales. Papeles del Psicólogo, 31(1), 34-45.

Sanz, M. (2008). Escala de Satisfacción Familiar (Fsfs. Family Satisfaction Scale). En Equipo EIF (Evaluación e Intervención Familiar), Manual de Instrumentos de Evalulación Familiar (pp. 69-77). Madrid: Editorial CCS

Sobrino, L. (2008). Niveles de satisfacción familiar y de comunicación entre padres e hijos. Avances en Psicología, 16(1), 109-137.

Stallman, H. M. (2010). Psychological distress in university students: A comparison with general population data. Australian Psychologist, 45(4), 249257. doi: 10.1080/00050067.2010.482109

Stephens, N. M., Fryberg, S. A., Markus, H. R., Johnson, C. S., \& Covarrubias, R. (2012). Unseen disadvantage: how American universities' focus on independence undermines the academic performance of first-generation college students. Journal of personality and social psychology, 102(6), 1178. doi: $10.1037 / \mathrm{a} 0027143$
Stover, J. B., de la Iglesia, G., Boubeta, A. R., \& Liporace, M. F. (2012). Academic Motivation Scale: adaptation and psychometric analyses for high school and college students. Psychology Research and Behavior Management, 5, 71-83. doi: 10.2147/PRBM.S33188

Timmerman, M. E., \& Lorenzo-Seva, U. (2011). Dimensionality Assessment of Ordered Polytomous Items with Parallel Analysis. PsychologicalMethods, 16, 209-220.

Turliuc, M. N., \& Buliga, D. (2014). Work-Family Conflict and Job and Family Satisfaction. The Mediating Role of Cognitions. Procedia-Social and Behavioral Sciences, 159, 105-109. doi: 10.1016/j. sbspro. 2014.12 .338

Widaman, K. F., \& Reise, S. P. (1997). Exploring the measurement invariance of psychological instruments: Applications in the substance use domain. En K. Bryant, M. Windle, \& S. West, The science of prevention: Methodological advances from alcohol and substance abuse research. (pp. 281-324). Los Angeles: American Psychological Association. 